\title{
Apprendre à écrire en anglais scientifique dans le secteur Lansad en master : quelles aides pour l'autonomisation des étudiants?
}

Sophie Bailly and Véronique Lemoine-Bresson

\section{OpenEdition}

Journals

Electronic version

URL: http://journals.openedition.org/ripes/2622

DOI: $10.4000 /$ ripes.2622

ISSN: 2076-8427

Publisher

Association internationale de pédagogie universitaire

\section{Electronic reference}

Sophie Bailly and Véronique Lemoine-Bresson, "Apprendre à écrire en anglais scientifique dans le secteur Lansad en master : quelles aides pour l'autonomisation des étudiants?", Revue internationale de pédagogie de l'enseignement supérieur [Online], 36(2) | 2020, Online since 21 September 2020, connection on 29 January 2021. URL: http://journals.openedition.org/ripes/2622 ; DOI: https://doi.org/ $10.4000 /$ ripes.2622

This text was automatically generated on 29 January 2021

Article L.111-1 du Code de la propriété intellectuelle. 


\title{
Apprendre à écrire en anglais scientifique dans le secteur Lansad en master : quelles aides pour l'autonomisation des étudiants?
}

\author{
Sophie Bailly and Véronique Lemoine-Bresson
}

\section{Introduction}

1 Les enseignements d'anglais en master à l'université française sont devenus obligatoires avec la réforme LMD (Licence, Master, Doctorat), sans doute en partie en raison de la place importante accordée actuellement à l'anglais comme langue de communication scientifique, dans un contexte de mondialisation auquel les études universitaires et la recherche scientifique n'échappent pas. Dans le cadre de la formation à la recherche, il est attendu des étudiants de master qu'ils puissent lire des textes de référence de leur spécialité scientifique en anglais et produire des écrits de genre scientifique dans leur discipline (mémoires, projets, articles, supports de communication orale) en français et, de plus en plus souvent, en anglais.

2 A l'Université de Lorraine (ci-après UL), les enseignements du master de didactique des langues étrangères et FLE (français langue étrangère) intègrent ce besoin de formation sous la forme d'un cours dédié à la lecture et à l'écriture de recherche dans leur discipline en anglais. L'une des caractéristiques du public de ce master, est qu'il présente généralement une forte hétérogénéité linguistique. Les étudiants proviennent de licences diverses: sciences du langage, lettres, langues et cultures étrangères, notamment d'anglais. Certains étudiants étrangers n'ont quasiment jamais appris l'anglais précédemment à leur arrivée en France. A cette diversité linguistique s'ajoute une diversité dans les aptitudes à lire et écrire des textes scientifiques y compris en français. Une autre caractéristique du public est qu'il a de fortes attentes de professionnalisation. Une minorité seulement des diplômés poursuivra des études en 
doctorat. Une autre partie des diplômés postulera comme enseignants de français langue étrangère (ou d'autres langues) dans des universités internationales ou dans le cadre de la formation en langue des adultes. En tenant compte de cette diversité des compétences et des besoins, il nous a semblé pertinent que le cours poursuive les objectifs suivants, dans une visée réflexive des contenus théoriques de leur discipline :

- amener les étudiants à progresser en autonomie dans la compétence langagière visée quelles que soient leurs compétences initiales, en appui sur un dispositif de type «apprentissage autodirigé avec soutien »

- amener les étudiants à construire de nouvelles connaissances théoriques disciplinaires;

- initier les étudiants à la recherche, notamment en termes de planification, d'auto-régulation et d'autonomisation.

3 Les modalités d'enseignement-apprentissage, inspirées des dispositifs de type système d'apprentissage autodirigé avec soutien (SAAS) en langues (Holec, 1988, 1998; Albero, 2000; Carré et Moisan 2002; Giveh et al., 2018), sont centrées sur l'aide à l'autonomisation des étudiants. Notre recherche vise à analyser les éléments d'aide extérieure qui sont proposés dans le cadre de ce cours et leurs effets sur les étudiants, à partir de questionnaires proposés en fin de cours.

\section{Aider à se former à écrire en anglais langue étrangère en master}

4 Avant d'aborder les travaux d'arrière-plan théorique qui sous-tendent notre étude, il nous semble utile de préciser que cette recherche s'inscrit dans le domaine de la didactique des langues en contexte Lansad (langues pour spécialistes d'autres disciplines). Bien qu'elle s'y apparente, elle ne relève pas des academic writing research, ni des second language writing research. Elle ne relève pas non plus des recherches en anglais de spécialité (ASP), ni des recherches en rédactologie, ni des recherches en didactique des écrits, ni des recherches en littéracies universitaires.

5 L'acronyme Lansad a été inventé en France par Michel Perrin au début des années 1990 pour rendre compte de la spécificité des besoins langagiers des étudiants universitaires qui ne sont pas des spécialistes en langues, mais en d'autres disciplines, et pour lesquels la langue anglaise s'impose comme élément de formation incontournable. Les étudiants qui relèvent de ce secteur Lansad constituent un public qui se distingue à la fois des étudiants spécialisés en études anglophones et des étudiants en mobilité dans un pays anglophone se formant en rédaction scientifique. Dans ce contexte l'anglais devient pour eux une langue seconde (plutôt qu'une langue étrangère) et les enseignants sont majoritairement nativophones de l'anglais, ce qui n'est pas le cas en secteur Lansad où les enseignants sont, en France, majoritairement francophones. Au niveau master, l'anglais est en général enseigné dans une visée instrumentale professionnelle en tant que langue additionnelle pour la recherche scientifique. Les aptitudes langagières enseignées sont le plus souvent la lecture de textes, la compréhension de communications scientifiques orales et écrites et la production scientifique orale et écrite, en anglais, dans le domaine disciplinaire de chaque master.

6 Pour l'aptitude de production écrite en langue étrangère, nombreux sont les travaux qui soulignent l'importance du temps réel dédié à la pratique de l'écrit. Dans cette partie nous expliquons pourquoi nous avons fait le choix d'une organisation 
pédagogique qui favorise l'autonomisation de l'apprentissage de l'écriture scientifique en anglais grâce à la mise en place de diverses modalités d'aide aux prises de décisions et aux actions des étudiants.

\subsection{L'écriture en anglais scientifique entre savoirs et savoir-faire}

7 Selon les domaines de recherche, l'écriture peut être envisagée comme un produit ou comme un processus. Nous l'envisageons quant à nous comme un savoir-faire à développer dans le cadre d'une formation institutionnelle à l'université. Pour Halté (1989) : « Le SE ${ }^{1}$ est un méta savoir-faire cognitif. Il organise, sous l'orientation donnée par le projet d'un scripteur, des $\mathrm{SF}^{2}$ disponibles, particuliers et complexes. Il se caractérise par un haut degré d'inventivité » (p. 8). L'écriture est considérée, depuis longtemps (Hayes et Flower, 1980), et de manière robuste semble-t-il, comme « une activité de résolution de problèmes qui entraine réflexion et prises de décision permanente» (Escorcia et Fenouillet, 2011, p. 57) mobilisant fortement la concentration et l'attention. Ce qui distingue principalement les scripteurs experts des novices est l'expérience, que nous définissons comme une combinaison de l'expérimentation et de sa mise à distance par la réflexion. Contrairement à l'expérimentation, l'expérience suppose un temps pour l'action et un temps pour la réflexivité. La réflexivité implique une modification de sa propre pensée et de sa propre action, comme conséquence d'une prise de conscience de ses connaissances implicites et d'un jugement sur ses pratiques à partir de nouveaux savoirs acquis -ou construitsau cours du processus formatif global de chaque individu (Ciekanski et al., 2016). Les experts scripteurs savent mieux s'auto-réguler et réviser leurs écrits parce qu'ils sont plus critiques grâce à des connaissances spécialisées sur le domaine traité, sur les usages de l'écrit du genre visé et sur les attentes du lectorat. En général, les étudiants qui réussissent dans leurs études semblent mobiliser également la connaissance de soi en tant qu'apprenants (méta-cognition) (Amadieu et Tricot 2015), même si Escorcia et Fenouillet (2011) relèvent que l'existence de liens positifs entre réussite et métacognition ne fait pas encore l'objet de consensus. En revanche, dans le domaine de l'écriture, des recherches «semblent s'accorder autour du rapport positif qui existe entre la performance et certaines opérations de planification, d'évaluation et de contrôle » (Escorcia et Fenouillet, 2011, p. 54-55), et entre le degré de compétence et le sentiment d'auto-efficacité (Pajares, 2003).

Des facteurs psychologiques liés au couple émotion-cognition influent également sur l'apprentissage et l'acquisition en général et du savoir écrire en particulier. Parmi ces facteurs nous citerons le sentiment d'auto-efficacité, croyance en ses capacités à réussir (Bandura, 1991) dont l'effet positif sur le processus d'écriture a été souligné entre autres par Pajares (2003), Van de Poel et Gasiorek (2012) et Escorcia et Fenouillet (2011). Amadieu et Tricot (2015) identifient d'autres facteurs de réussite : les buts de maitrise, qui impliquent « une évaluation auto-référencée de l'étudiant, organisée autour de la progression personnelle » (Amadieu et Tricot, 2015, p. 3), la perception de l'utilité et de la valeur de la tâche, le contrôle sur la tâche ou tout au moins le sentiment de contrôle. Ces auteurs reconnaissent tous l'importance de l'autorégulation dans la réussite des étudiants. La capacité d'autorégulation implique de pouvoir gérer, mobiliser et contrôler ses connaissances, ses émotions, et ses comportements. 


\subsection{Aider l'acquisition d'un savoir-faire d'écriture universitaire en anglais}

9 La conception du dispositif proposé aux étudiants du master Didactique des langues/fle de l'UL repose en partie sur le principe suivant, établi par Holec en 1998: «L'acquisition de savoir-faire est une acquisition d'expérience, et non plus de connaissances, et l'expérience s'acquiert par la pratique. Le seul apprentissage qui puisse mener à une telle acquisition est donc un apprentissage qui met en situation de faire ce que l'on veut être capable de faire " (Holec, 1998, p. 221). Cette vision prend sa source dans les travaux des années 1960 qui «font de l'individu et de son expérience propre le foyer d'un processus d'apprentissage » (Lochard, 2007, p. 83). Elle se conjugue dans des variations autour de l'auto qui prennent la forme d'autogestion, d'autonomie et d'autorégulation dans un contexte donné. Ainsi, nos modalités d'enseignement tiennent compte de ces éléments, mais également de ceux apportés par Howes (1999), Street (1999) ou encore Johns (1997) qui considèrent parmi les premiers que l'écrit peut difficilement être enseigné comme un ensemble composé de compétences dissociables que l'on peut apprendre et acquérir séparément pour ensuite les appliquer à tous les contextes d'écriture. A l'inverse, une approche qui consisterait à se contenter d'exposer des apprenants à de "bons " modèles n'est pas non plus satisfaisante, puisque «students do not learn by osmosis" (Van de Poel et Gasiorek, 2012, p. 296). Pour améliorer la réussite dans l'écriture universitaire (en français) Escorcia et Fenouillet (2012) estiment qu' «il est davantage utile d'aider le sujet à adapter ses stratégies personnelles aux conditions de la tâche et à ses habilités personnelles, plutôt que lui apprendre des méthodes générales censées favoriser les performances en écriture de tous les scripteurs sans distinction» (p. 69). Une approche pédagogique centrée sur la réussite ne peut pas se contenter d'enseigner des contenus théoriques sur l'écriture en anglais. Elle devrait prendre en compte le rapport à l'écriture (Barré de Miniac, 2001) des étudiants et s'adresser à leurs connaissances, leur conscience et leurs émotions (Van de Poel et Gasoriek, 2012). Les connaissances à mobiliser et à gérer dans l'acte d'écriture concernent les genres d'écrits à produire, les normes par lesquelles ces genres sont évalués, les contenus à écrire, et le soi écrivant. La conscience des étudiants est mobilisée à plusieurs niveaux : (i) la conscience des buts à atteindre et des moyens d'y parvenir; et (ii) la conscience de leurs propres connaissances et stratégies, dont ils doivent évaluer l'efficacité pour atteindre le but fixé, agissant ainsi de manière autonome dans la gestion de leur écrit (Escorcia et Fenouillet, 2012). Pour aider les étudiants à auto-réguler les différentes tâches inhérentes à l'écriture en anglais, Xiao et Yang $(2019$, p. 41) préconisent de rendre explicites les critères d'évaluation et d'aider les étudiants à apprécier positivement et négativement leur propre travail en leur donnant un feed-back formatif qu'ils puissent comprendre. Agir sur la conscience des étudiants peut se faire à deux niveaux. Tout d'abord, il s'agit de développer la conscience de soi apprenant auteur (ou scripteur) en réfléchissant à ses acquis et lacunes, ses forces et faiblesses, ses stratégies personnelles efficaces, son parcours et ses responsabilités, etc. Ensuite se joue la conscience des attentes et des enjeux de formation, fortement reliée aux notions d'évaluation et d'auto-évaluation, aux normes et à la compréhension des critères. Les prises de conscience peuvent être soutenues par un retour sur l'expérience dans une activité réflexive, soit en répondant à des questionnaires spécifiques, soit en tenant un journal de bord (Chateau et Zumbhil, 2010), soit dans un dialogue avec l'enseignant -tel que décrit par exemple par Gremmo 
(1995), Bailly (1995) et Ciekanski (2005) sous le terme de conseil en apprentissage de langue.

10 Finalement, des émotions sont engagées dans l'acte d'écrire, sous forme positive comme la motivation, ou négative, comme l'anxiété. L'apprentissage de l'écriture scientifique représente une transformation de l'identité sociale de l'étudiant qui peut plus ou moins bien vivre et accepter d'assumer une identité d'auteur (Coffin et al., 2003). C'est le rôle de l'encadrement pédagogique de prendre sa part de responsabilité et d'accompagner cette transformation (Macrae, 1997; Blašková, 2014). Les étudiants semblent d'ailleurs avoir des attentes précises quant aux rôles et attitudes des enseignants. Ils espèrent de ces derniers un rapport harmonieux, une posture d'encouragement, un comportement équitable et de la disponibilité (Amadieu et Tricot 2015). Le dialogue enseignantapprenant est une modalité de médiation qui peut créer de bonnes conditions psychologiques et soutenir la motivation (Xiao et Yang, 2019). Pour prévenir ou réduire l'anxiété qui peut surgir lorsque les étudiants doivent lire des références de leur spécialité scientifique en anglais et produire des écrits de genre scientifique dans leur discipline en anglais, l'enseignant peut proposer un accompagnement individuel et adopter une posture d'écoute et de soutien encourageant.

11 Des conditions matérielles minimales externes à l'apprenant sont finalement nécessaires pour que l'apprentissage du savoir-écrire se déroule positivement et débouche sur des acquisitions. L'une de ces conditions est le temps (ou des moments) pour écrire. Il faut également disposer d'un espace calme, confortable et pratique, propice à la réflexion individuelle et collective, à la recherche d'informations et à la rédaction. Enfin, il n'y a pas d'écriture possible sans outils appropriés aux tâches à accomplir. De nos jours l'activité d'écriture scientifique en anglais peut s'appuyer sur de nombreux outils et applications numériques accessibles en ligne: des bases de données bibliographiques et textuelles, des concordanciers et corpus, des dictionnaires en ligne, des traducteurs automatiques, de plus en plus performants, des détecteurs de plagiat, des applications de gestion des références bibliographiques, des outils de traitements de textes, des assistants de modélisation graphique, des correcteurs orthographiques et grammaticaux, etc. Ajoutons, pour fermer non définitivement cette liste, les environnements numériques de travail et de formation -dont la plateforme Moodle, un LMS (learning management system) répandu dans les universités et en usage à l'UL sous le nom d'Arche ${ }^{3}$, qui, selon leur configuration peuvent aider les étudiants à gérer leur travail universitaire à distance.

Nous retenons de ces travaux que, pour aider les étudiants à développer leur savoirfaire en lecture et écriture de genre scientifique en autonomie, l'organisation pédagogique doit à minima respecter les conditions suivantes :

- la mise en situation de faire ce qu'il faut apprendre à faire, ou apprentissage expérientiel (Giveh et al., 2018);

- la mise à disposition de ressources accessibles, autosuffisantes et adaptables (Carette et Holec, 1995);

- l'organisation d'espaces-temps de réflexion et de réflexivité;

- des interactions apprenant-enseignant selon diverses modalités d'accompagnement et de soutien.

Ces conditions induisent nécessairement un changement des rôles des apprenants et des enseignants vers plus d'activité pour les premiers et plus d'écoute, de soutien et de réactivité (feed-back) pour les seconds (Bailly et Ciekanski, 2004). 


\section{Organiser un système d'aide à l'autonomisation pour la formation à l'écriture scientifique en anglais en master : pourquoi? comment?}

Pour situer la recherche nous présentons de manière détaillée les objectifs de la formation, son organisation et déroulement en discutant notamment des rôles apprenant et enseignant, ainsi que l'environnement numérique d'apprentissage sous ses aspects de fontionnement et de contenus. Nous abordons également les spécificités de l'accompagnement et de l'évaluation dans les diverses modalités d'aide mises en œuvre.

\subsection{Objectifs de formation}

La conception de l'organisation répond à un double objectif : amener les étudiants à, d'une part, acquérir un savoir écrire et à développer leur expérience en rédaction scientifique en anglais; et d'autre part, à devenir plus autonomes dans les décisions à prendre et actions liées à leurs apprentissages. Cela se fait progressivement. En master 1 , le premier semestre a pour objectif d'améliorer les habiletés en lecture de textes scientifiques en anglais. Au deuxième semestre l'objectif de rédiger un texte plus long et en anglais, toujours à partir d'un corpus d'articles authentiques (Abrams, 2019). Ce texte doit répondre aux critères du genre textuel 'cadrage théorique' ou 'revue de littérature' d'un article scientifique en sciences humaines et sociales, en anglais. Les étudiants rédigent leur production en anglais, à partir d'une question de recherche ou d'un centre d'intérêt qu'ils choisissent et construisent en lien avec leur discipline. En master 2, l'objectif visé est la présentation d'une communication scientifique orale. Au quatrième semestre les étudiants sont en stage. Notre étude porte sur le deuxième semestre du master 1 , soit le moment de l'entrée en écriture scientifique en anglais.

\subsection{Déroulement et organisation}

Le cours d'anglais scientifique, son nom officiel dans la maquette, occupe un volume de 24 heures de cours de travaux dirigés par semestre en présentiel. Le cours affiche 1 $\mathrm{ECTS}^{4}$, ce qui amène le temps total théorique de travail étudiant attendu à entre à environ 36 heures par semestre (comprenant 24 heures de temps de présence en cours). Les heures en présentiel se déroulent dans une salle informatique du campus, pour rendre possible l'accès au numérique à tous les étudiants qui ont à intégrer la dimension technologique du savoir-écrire en contexte scientifique. Tous les étudiants n'ont pas forcément accès depuis chez eux à des outils numériques professionnels voire à des connexions internet de qualité, il est indispensable d'égaliser les chances de départ. Les 12 heures de travail personnel additionnel sont gérées par les étudiants eux-mêmes en termes de rythme, durée et lieu. Des salles informatiques en accès libres sont disponibles sur le campus pour les moments de travail en dehors du créneau de cours. Les étudiants peuvent en outre retrouver leurs documents de travail depuis n'importe quel ordinateur connecté à l'espace numérique de travail de l'UL, y compris depuis chez eux. Cette organisation matérielle est la même pour les trois semestres de cours. Seul l'objectif, et donc les ressources, varient de semestre en semestre. Les 
scénarios propres aux trois cours sont présentés et expliqués sur une page Arche dédiée au cours. La première séance de cours présentiel est consacrée à une présentation des attendus du cours (le projet d'écriture), des critères d'évaluation (le cours doit donner lieu à une note) et des modalités de travail, et à une clarification des rôles étudiant et enseignant dans l'organisation. La page Arche est projetée et commentée en séance et les étudiants procèdent à des essais pratiques des outils à utiliser. Lors des séances suivantes, les étudiants commencent concrètement à réfléchir à et à réaliser leur projet d'écriture, en solo ou en duo. L'organisation prévoit une répartition spécifique des rôles selon le statut étudiant ou enseignant. Nous la présentons dans le tableau 1 ci-dessous.

$\operatorname{Tableau}^{\circ} 1$. Répartition des rôles apprenant et enseignant

\begin{tabular}{|c|c|}
\hline Tâches de l'étudiant & Tâches de l'enseignante \\
\hline $\begin{array}{l}\text { Prendre connaissance du scénario } \\
\text { pédagogique accessible dès le début du } \\
\text { cours sur Arche. } \\
\text { Définir librement }{ }^{5} \text { un sujet de recherche, } \\
\text { à partir de leurs centres d'intérêt, des } \\
\text { contenus de la formation, ou d'une } \\
\text { question-problème constatée sur le } \\
\text { terrain lors d'un stage de découverte ou } \\
\text { de pratique. } \\
\text { Choisir des ressources et des outils, les } \\
\text { utiliser et les évaluer en fonction de leurs } \\
\text { besoins. } \\
\text { Faire des recherches en bibliothèque ou } \\
\text { en ligne pour recueillir un corpus de } \\
\text { textes scientifiques en rapport avec le } \\
\text { sujet de la recherche. } \\
\text { Rédiger le texte attendu en anglais, en } \\
\text { respectant les consignes d'écriture et en } \\
\text { ayant recours à toutes les aides possibles } \\
\text { y compris celle de relecteurs de son } \\
\text { choix, ou celle des outils numériques } \\
\text { d'aide à l'écriture en anglais (traducteurs, } \\
\text { dictionnaires, corpus, et concordanciers). } \\
\text { Analyser régulièrement son travail et } \\
\text { rendre compte de ses activités et de ses } \\
\text { réflexions dans un carnet de bord en } \\
\text { ligne, sorte de journal d'écriture de } \\
\text { recherche, qui peut être lu par } \\
\text { l'enseignante. }\end{array}$ & $\begin{array}{l}\text { En amont du cours: curation de contenus et de } \\
\text { ressources tels que des connaissances dans la discipline } \\
\text { et sur l'écriture scientifique en anglais; des outils de } \\
\text { rédaction et de réflexivité; des exemples d'articles et de } \\
\text { sujets de recherche. } \\
\text { Pendant la durée du cours et à distance : améliorer et } \\
\text { adapter les contenus du cours sur Arche lorsque cela } \\
\text { est nécessaire; suivre le travail des étudiants via leur } \\
\text { carnet de bord, en leur fournissant conseils, feed-back } \\
\text { formatif ou encouragements et propositions d'aide. } \\
\text { Apprécier les modalités de travail de l'étudiant et aider } \\
\text { chaque étudiant à résoudre les problèmes rencontrés } \\
\text { par le biais de commentaires personnalisés. } \\
\text { En séance: conseiller individuellement et } \\
\text { collectivement sur le choix du sujet de recherche, les } \\
\text { méthodes de travail, et toute autre question des } \\
\text { étudiants. } \\
\text { En fin de cours : évaluer les productions finales et } \\
\text { attribuer une note et un feed-back correctif. } \\
\text { al }\end{array}$ \\
\hline
\end{tabular}

17 Dans cette organisation les étudiants sont immédiatement mis en situation d'auteur professionnel novice qui a un temps limité (dans notre cas une trentaine d'heures) pour produire un texte de 5 pages environ (2500 mots) de type cadre théorique ou littérature review d'un article scientifique. Les responsabilités liées à l'auto-régulation sont 
partagées entre les étudiants et l'enseignante qui assume sa part en tant que conceptrice, décideuse (objectifs, buts à atteindre), animatrice et accompagnatrice. Ce point sera développé en section 3.4.

\subsection{L'environnement numérique de travail} organisées selon plusieurs thèmes: (1) l'évaluation et les modalités de contrôle des connaissances; (2) les connaissances à mobiliser dans la tâche d'écriture, et à construire; les outils numériques d'aide à la rédaction en anglais; (3) les conseils de l'enseignante pour aborder et réussir la tâche demandée. Une dernière section (4) rassemble les outils de la communication individuelle ou collective entre l'enseignante et les étudiantes.

\section{Section MCC (modalités de contrôle des connaissances)}

19 Un descriptif des MCC (modalités de contrôle des connaissances démarre la page, ce qui permet dès la première séance de travail de clarifier les buts à atteindre. Cette première section du cours présente immédiatement les attendus et barèmes de notation. Elle contient une description détaillée des pièces à fournir pour le dossier et des critères d'appréciation qui seront appliqués pour l'évaluation finale. (Annexe 1). Cette section se complète d'un lien vers un carnet de bord (en fait un wiki individuel intégré à Arche) qui sert d'outil de suivi et de communication entre l'enseignante et chaque étudiant.

\section{Section Connaissances}

Dans cette section sont rassemblées des ressources pour soutenir la construction de connaissances sur le genre de texte à produire, et pour signaler les points de vigilance du savoir-faire d'écriture en anglais scientifique qui est attendu dans le cadre de ce cours. Elle présente des textes pédagogiques rédigés par l'enseignante en mode page sur Arche (descriptions, consignes, conseils, informations, annonces etc.), ainsi que des publications d'autres auteurs (par exemple, Pasek, 2012; Coleman, 2014; van Enka et Power, 20176), qui sont accessibles en ligne par des liens hyper-textes ou en version PDF. Ces ressources en français et en anglais apportent des éléments de réponses à des questions comme: qu'est-ce qu'un article scientifique en anglais? Qu'est-ce qu'un cadrage théorique ou un état de l'art dans le domaine visé en anglais? Comment est-il organisé, structuré? Qu'est-ce qu'un abstract? Quelles sont les règles à respecter pour réussir la tâche?

21 Les ressources visant les aspects de langue anglaise se composent de documents authentiques $^{7}$ et didactiques en lien avec l'objectif spécifique de l'anglais écrit en sciences sociales. Elles se présentent pour la plupart sous forme de liens vers des guides de rédaction scientifique, des exercices en lignes, des banques de phrases spécifiques à la rédaction scientifique en anglais, des exemples du genre visé, des cours d'argumentation écrite en anglais scientifiques, ou encore des conseils de rédaction d'experts en rédaction scientifique en anglais ${ }^{8}$. Ces ressources ont été sélectionnées par l'enseignante sur la base des critères d'accessibilité, adaptabilité et d'auto-suffisance (Carette et Holec, 1995), c'est-à-dire que l'étudiant est en mesure de les consulter

Revue internationale de pédagogie de l'enseignement supérieur, 36(2) | 2020 
facilement à tout moment, d'en faire usage selon ses besoins propres et sans aide supplémentaire extérieure. Elles sont présentées avec un court commentaire de l'enseignante qui est censé soutenir la décision de l'étudiant de s'y intéresser plus avant ou pas, en fonction de ses besoins ou des problèmes en cours de résolution.

\section{Section Outils d'assistance à la rédaction en anglais}

Cette section rassemble une sélection d'outils linguistiques tels que des traducteurs automatiques, des corpus et concordanciers en ligne, des dictionnaires en ligne. Elle comprend également des outils d'assistance à la recherche scientifique en ligne : bases de données de revues en ligne; gestionnaire de références bibliographiques (Zotéro).

\section{Section Conseils}

23 La section Conseils s'adresse au développement de la conscience et de la métacognition des étudiants, et à leur auto-régulation, facteurs dont nous avons vu l'importance plus haut (Cf point 2.2.). Ses contenus sont censés aider les étudiants à valider ou expérimenter des stratégies cognitives, affectives et sociales et à prendre conscience des différentes tâches qu'ils auront à organiser et à réaliser. Ces conseils sont disponibles comme le reste du cours dès le démarrage du semestre, et leur présentation est faite dès la première séance. Les étudiants sont censés évaluer par eux-mêmes l'utilité et la pertinence de ces conseils selon l'étape de développement de leurs compétences et les problèmes qui se posent à eux en cours de leur recherche ou de leur rédaction éventuellement aidés en cela par l'enseignante. Il est attendu d'eux qu'ils se conduisent comme des apprenant autorégulés, c'est-à-dire qu'ils s'investissent dans cette phase d'apprentissage de manière métacognitive, motivationnelle et comportementale (Pintrich, 2004). Les conseils portent notamment sur la préparation du projet, la méthodologie de rédaction, les usages en matière de citation des sources, les méthodes de révision, d'auto-correction et d'auto-évaluation. Dans l'exemple 1 (Annexe 2), le conseil vise à remettre en mémoire les différentes étapes de la production d'une problématique et suggére différentes manières de faire. L'exemple 2 (Annexe 2) contient des conseils méthodologiques pour la phase de rédactionrelecture-révision. Outre ces documents de soutien à l'autonomie accessibles à tout moment (Bailly, 1995), les étudiants trouvent dans cette section un questionnaire d'auto-analyse de leurs stratégies de gestion de leur projet d'écriture.

\section{Section Communication}

Dans cette section se trouvent des instruments de communication asynchrone collective et individuelle : un forum des nouvelles générales et un forum de questionsréponses sur les MCC. Elle comprend aussi le carnet de bord individuel de chaque étudiant. La compréhension du statut et de la fonction du carnet de bord est diverse selon les étudiants et suscite des réactions positives, ou négatives qui dans certains cas évoluent avec le temps. En cas de doute sur la finalité du carnet de bord et des contenus attendus, les étudiants sont guidés par une consigne et des questions placées en exergue du carnet de bord (Annexe 5) et auxquelles ils peuvent se référer en cas de panne d'inspiration. Une fonction Commentaire intégrée au wiki-Carnet de bord permet d'écrire un message de réaction à la lecture du carnet de bord, validations, encouragements, suggestions, questions, etc. C'est aussi dans ce carnet de bord que les 
étudiants remettent leur dossier final et que l'enseignante dépose le dossier évalué, annoté et corrigé.

\subsection{L'accompagnement}

25 La notion d'accompagnement, que Paul (2004) qualifie de posture professionnelle spécifique, implique de "conduire, guider et escorter» des personnes dans une entreprise "risquée, problématique et éprouvante " (Paul, 2004, p. 68-69). Par risquée, il faut comprendre que l'entreprise « expose à des dangers éventuels, hasards ou aléas; par là même ne dépendant pas exclusivement de la volonté des protagonistes, elle est sans garantie quant au résultat» (Paul, 2004, p. 68). L'atteinte des objectifs attendus pour le cours d'anglais scientifique n'est pas gagnée d'avance. Le succès peut dépendre de la facilité à trouver un sujet ou des sources fiables, ou encore à surmonter les difficultés linguistiques et épistémiques ou liées à la gestion et aux émotions. De même, des difficultés personnelles de tous ordres peuvent survenir et mettre en danger le bon déroulement du projet. Pour Paul (2004), une entreprise problématique "réfère à une question qui prête à discussion ou délibération, question exigeant une décision alors même qu'on ne possède pas, par définition, les moyens d'évaluer celle-ci » (p. 68). L'écriture scientifique en anglais est doublement problématique pour les étudiants novices en ce domaine. Premièrement, écrire en langue étrangère est processus qui implique des prises de décisions constantes et la gestion en parallèle de savoir-faire de divers ordres. Deuxièmement, la recherche scientifique qui constitue le contenu thématique du texte à écrire, répond également à ces critères de discussion, délibération et décision. En tant que processus de modification identitaire (Coffin, et al., 2003), devenir auteur scientifique, de surcroit en anglais, est une entreprise éprouvante pour les étudiants, dans la mesure où : « quelle que soit la décision prise, il s'agit de la mettre à l'épreuve et, inévitablement, d'être soi-même éprouvé par rapport au choix effectué puisque, s'il s'agit d'aller, de se mettre en mouvement, de déterminer une direction, il n'est pas dit qu'il faille aller n'importe comment mais selon une certaine ligne de conduite » (Paul, 2004, p. 69).

Pour leur projet d'écriture d'une recherche, les étudiants ont à prendre des décisions sur le choix d'un sujet, de sources, d'une méthodologie personnelle, d'un calendrier ou encore sur le fait de conserver leur stratégie ou d'en changer. Ils connaissent leur objectif (voir le point MCC en 3.3.) et doivent décider d'une ligne de conduite pour l'atteindre, voire décider s'il font ou non une demande d'aide. En ce sens, la double entreprise d'écrire un texte scientifique en anglais et d'apprendre à le faire par soimême, répond à ce qualitatif d'éprouvante.

Pour ces raisons, dans notre organisation, l'enseignement présentiel laisse une part importante à l'accompagnement individuel. Il ne s'agit plus d'enseigner de manière collective, des contenus généraux sur les méthodes de rédaction scientifique ou des règles linguistiques générales, mais bien de fournir de l'aide personnalisée pour apprendre par soi-même et surmonter les difficultés de tous ordres qui peuvent se présenter à l'étudiant pour qu'il garde le contrôle sur la situation. Le type d'accompagnement que nous proposons prend en grande part sa source dans le conseil en apprentissage autodirigé de langues théorisé par Gremmo (1995) et Ciekanski (2005). Ce type de conseil a lieu en présenciel lors d'un entretien individuel et est réactif aux demandes ou analyses de l'apprenant. Une autre forme d'accompagnement réactif se 
fait en mode asynchrone, lorsqu'il s'agit de répondre à une demande exprimée par écrit dans l'outil carnet de bord, via sa fonction commentaire. Finalement, l'accompagnement prend également la forme d'un feedback correctif sur la langue et d'un feedback formatif sur le projet, via des commentaires, des explications, des conseils adressés personnellement à chaque étudiant. Nous développons cet aspect ciaprès.

\subsection{Evaluation, auto-évaluation et co-évaluation}

Pour être congruent avec l'objectif d'autonomisation de l'apprentissage, qui implique de prendre une part active à tous les actes de l'apprentissage, y compris l'évaluation (Holec, 1981, 1998), l'acte d'évaluation est partagé entre les étudiants et l'enseignante. Nous décrivons ici les modalités des évaluations sommative, formative et corrective de ce cours.

\section{Auto et co-évaluation sommative}

La contrainte institutionnalisée de fournir une note chiffrée, autrement dit l'évaluation sommative n'est guère compatible avec les visées d'une organisation autonomisante qui agit sur plusieurs fronts dont une seule note ne saurait rendre compte. Malgré ce paradoxe, pour répondre néanmoins à cette contrainte institutionnelle de notation chiffrée tout en gardant un minimum de cohérence pédagogique, la note finale se construit selon deux principes proposés à discussion aux étudiants en début de semestre. Premièrement, une part de la note finale vaut pour le texte en anglais selon un barème connu des étudiants (dans la section MCC) et l'autre part vaut pour l'autoévaluation de l'acquisition du savoir faire visé, telle qu'elle peut s'apprécier à partir de la lecture du carnet de bord. Deuxièmement, la note finale résulte d'une négociation entre une appréciation chiffrée de son travail par l'étudiant (auto-notation) et la note proposée par l'enseignante (hétéro-notation), qui module et signale de possibles effets d'auto-sur-estimation ou d'auto-sous-estimation. Le bilan de cette négociation et la décision finale de l'enseignante sont explicités sur le carnet de bord (voir exemple en Annexe 3).

\section{L'(auto)-évaluation formative : un dialogue intra et interpersonnel}

Dans le cadre des dispositifs d'apprentissage autodirigé en contexte universitaire, le carnet de bord est souvent proposé comme instrument de réflexivité pour l'autoévaluation et l'amélioration de l'activité (Chateau et Zumbhil, 2010). Le temps de l'écriture du carnet de bord se veut un temps pris pour réfléchir sur le soi-auteur-enformation, en faisant le bilan des actes et des décisions, et en se projetant dans les futurs actes et décisions (voir deux exemples en Annexe 4). Le carnet de bord garde également la trace du processus de recherche et d'écriture lorsque les étudiants l'instrumentalisent en tant que journal de recherche. En le rédigeant et en le relisant, ils peuvent constater le chemin parcouru et en tirer des conclusions pour eux-mêmes. L'auto-évaluation formative prend aussi la forme d'un questionnaire en ligne, et accessible à tout moment du cours, sur les difficultés et les stratégies de résolution de problèmes. Il comprend des questions ouvertes et des questions fermées à choix multiples (Annexe 6) portant sur leurs aptitudes, attitudes, usages, comportements, 
savoir-faire, connaissances, stratégies. Ces questions peuvent leur servir d'indicateurs précis pour évaluer leurs savoir-faire ${ }^{10}$.

Outre ces supports d'un dialogue intrapersonnel, l'évaluation formative prend aussi la forme d'un dialogue interpersonnel continu, entre chaque étudiant et l'enseignante. Il $\mathrm{y}$ a trois périodes de suivi. En début de semestre, le premier suivi a lieu en troisième semaine de cours. L'enseignante vérifie si chacun s'est emparé du carnet de bord, et où en est chacun dans la phase d'élaboration du projet d'écriture: choix du sujet, de revues, d'articles, d'outil, notes en français ou en anglais, questions pour l'enseignante, etc. En milieu de semestre, il s'agit d'apprécier la progression du projet d'écriture, dans sa dimension processuelle et textuelle et de fournir un feed-back portant sur les productions intermédiaires en anglais et sur la gestion du processus. Ce feed-back est l'occasion d'encourager, de proposer de l'aide, de répondre à des questions ou de donner des conseils pour la suite. Lorsqu'un texte intermédiaire est rédigé en anglais par l'étudiant dans son carnet de bord, un avis sur la qualité formelle est donné si nécessaire ou si demandé. Les premier et deuxième feed-back de suivi se font de deux manières possibles : directement dans le carnet de bord de l'étudiant par la fonction "Modifier» du wiki et par un ajout de commentaire ou de question. Cet écrit de l'enseignante est coloré de sorte à bien se distinguer du texte étudiant généralement en noir sur blanc. Le troisième et dernier moment de feed-back se situe en fin de parcours une fois les travaux remis. L'enseignante rédige un bilan final et explicite sa décision de note finale afin d'aider l'étudiant à la comprendre. Les étudiants disent apprécier ce bilan car selon eux, il donne du sens à l'évaluation (pour des exemples de ces différents feed-back voir Annexe 7). Des exemples de feed-back formatif des années précédentes ont été ajoutés comme ressources asynchrones pour l'auto-évaluation sur le cours en ligne lors de la troisième année du cours.

\section{Auto et co-évaluation corrective}

Le feed-back correctif sur la production en anglais est fait en surlignant en jaune des aspects linguistiques des textes intermédiaires en anglais qui seraient à vérifier par l'étudiant, ou par des questions et remarques par la fonction " Nouveau commentaire " de l'onglet 'Révision' sur word. Nous essayons de ne pas fournir la solution de manière systématique aux étudiants mais plutôt de les aider à s'auto-corriger.

\subsection{Synthèse du système d'aide proposé}

33 Nous synthétisons ci-dessous sous forme de tableau une analyse des aides proposées selon leur visée (à quel comportement à auto-réguler s'adressent-elles?), les types d'outils proposés et leurs fonctions dans le processus d'autonomisation.

Tableau 2. Les aides : analyse des visées, outils et fonctions

\begin{tabular}{|l|l|l|}
\hline Visées de l'aide & Outils de l'aide & Fonctions de l'aide \\
\hline
\end{tabular}




\begin{tabular}{|c|c|c|}
\hline $\begin{array}{l}\text { La connaissance des attentes } \\
\text { sur le genre à produire et sur } \\
\text { le thème choisi pour le } \\
\text { projet d'écriture }\end{array}$ & $\begin{array}{l}\text { Cours présentiel (dont un } \\
\text { cours spécifique sur } \\
\text { l'utilisation des corpus) et } \\
\text { cours en ligne } \\
\text { Contenus en ligne sur le type } \\
\text { de texte à produire et sur la } \\
\text { conduite du processus } \\
\text { Carnet de bord en ligne } \\
\text { Hyperliens vers les bases de } \\
\text { données de revues en ligne }\end{array}$ & $\begin{array}{l}\text { Clarifier la nature de l'objet } \\
\text { langagier à produire, la nature du } \\
\text { processus de rédaction et des } \\
\text { décisions qu'il implique } \\
\text { Chercher des idées et des contenus } \\
\text { scientifiques pour le projet } \\
\text { d'écriture } \\
\text { Construire et structurer des } \\
\text { connaissances par la prise de notes, } \\
\text { l'ébauche de plans ou de cartes } \\
\text { mentales, la rédaction de synthèses }\end{array}$ \\
\hline $\begin{array}{l}\text { Les savoir-faire } \\
\text { technologiques, } \\
\text { linguistiques, cognitifs et } \\
\text { linguistiques }\end{array}$ & $\begin{array}{l}\text { Outils numériques } \\
\text { d'assistance à la rédaction } \\
\text { Outils numériques propres au } \\
\text { métier d'étudiant chercheur } \\
\text { (Cf section Outils du cours en } \\
\text { ligne) }\end{array}$ & $\begin{array}{l}\text { Pratique de différents outils de } \\
\text { travail numériques dans l'acte } \\
\text { d'écriture de recherche } \\
\text { Pratique de l'auto-correction } \\
\text { Pratique de la rédaction de } \\
\text { recherche assistée par le numérique }\end{array}$ \\
\hline $\begin{array}{l}\text { La conscience: } \\
\text { de soi et de l'autre dans le } \\
\text { processus; } \\
\text { de ses stratégies et } \\
\text { responsabilités }\end{array}$ & $\begin{array}{l}\text { Carnet de bord en ligne } \\
\text { Interactions avec } \\
\text { l'enseignante } \\
\text { Questionnaires de soutien à la } \\
\text { réflexivité }\end{array}$ & $\begin{array}{l}\text { Utilisation de l'écriture réflexive } \\
\text { comme un instrument de prise de } \\
\text { conscience } \\
\text { Réfléchir à son processus d'écriture } \\
\text { et l'évaluer }\end{array}$ \\
\hline $\begin{array}{l}\text { Les émotions: } \\
\text { motivation, stratégies } \\
\text { gestion du stress, }\end{array}$ & $\begin{array}{l}\text { Entretiens de conseil en } \\
\text { présentiel et conseil en ligne } \\
\text { asynchrone et à distance } \\
\text { Accompagnement } \\
\text { personnalisé } \\
\text { Carnet de bord }\end{array}$ & $\begin{array}{l}\text { Réduction de l'anxiété et } \\
\text { augmentation du sentiment d'auto- } \\
\text { efficacité, de la confiance en soi et } \\
\text { en ses capacités d'auto-régulation et } \\
\text { d'auto-évaluation }\end{array}$ \\
\hline
\end{tabular}

Pour cloturer cette description de l'organisation, nous précisons qu'elle a donné lieu à des évaluations par chaque promotion d'étudiants depuis sa mise en place en 2014. Les retours obtenus ont induit quelques modifications et améliorations du cours en ligne et des modalités d'évaluation.

\section{Méthodologie}

Pour cette recherche, nous nous demandons quels sont les effets des aides apportées par le cours sur les savoir-faire développés et la mobilisation des connaissances. L'étude que nous présentons correspond à une phase d'évaluation du dispositif menée en 2015 avec pour objectif de recueillir un feed-back étudiant sur les modalités du cours. Cette année-là l'effectif du master 1 était composé de 13 étudiantes réparties en 8 francophones natives dont 3 issues d'une licence d'anglais, 3 sinophones, 1 arabophone et 1 farsiphone. En fin de semestre elles ont répondu à un questionnaire en ligne (voir Annexe 8) présenté comme une évaluation du cours en vue de son amélioration. Ce 
questionnaire, non obligatoire, invite les étudiants à revenir sur leur parcours et à analyser leurs éventuels progrès et le rôle joué par les aides proposées. Les questions portent sur leur compétence préalable au cours; sur leur perception du cours et des aides proposées; sur leur progression et leurs difficultés. D'un format assez court pour inciter à répondre, il se présente sous forme d'échelles de Likert et de questions ouvertes pour l'ajout de commentaires additionnels facultatifs. Toutes les étudiantes ont répondu à ce questionnaire. Les réponses ne sont pas anonymes, ce qui peut induire des biais liés à la sincérité des réponses.

Nous avons analysé les scores des échelles de Likert et les contenus des commentaires écrits à l'aune de la notion d'aide pour induire ce que les réponses indiquent (en bosse), et ce qu'elles n'indiquent pas (en creux) au sujet des effets - voire de l'efficience - et des limites du système d'aide proposé. Nous n'avons pas établi de grille figée mais avons plutôt laissé notre lecture des résultats chiffrés et des commentaires faire émerger des thèmes en rapport avec le savoir-faire et les connaissances en écriture d'anglais scientifique comme langue étrangère.

\section{Analyses et Résultats}

Nous présentons les réponses des étudiants à des questions concernant l'évaluation globale du cours, l'évolution de leur sentiment de compétence en écriture scientifique en anglais, l'évaluation des ressources proposées comme aides et les difficultés les plus importantes rencontrées. Nous analysons, à travers ces réponses, les effets de l'aide proposée sous cinq aspects : aide à la prise de conscience des connaissances déjà-là; aide à l'implication dans la tâche et l'accrochage; aide à la conscientisation du développement de compétences articulées à des savoirs; et aide à une approche variée des apprentissages; et, pour finir, les améliorations à apporter au système d'aide.

\subsection{Aide à la prise de conscience des connaissances préalables à la formation, ou déjà-la}

Les réponses des étudiants à la question "Décrivez ou évaluez brièvement vos compétences en rédaction scientifique en anglais avant le début du cours » font émerger des thématiques de niveaux en langues, d'une part des niveaux de compétences et d'autre part, des niveaux de référence habituellement utilisés dans le domaine, et notamment par le CECRL (cadre européen commun de référence pour les langues). Les analyses des commentaires confirment l'hétérogénéité linguistique en anglais. Ces déclarations sur les compétences présentes en début de cours sont intéressantes dans le processus d'autorégulation, car elles devraient permettre aux étudiants de prendre conscience de leurs connaissances en anglais et en écriture scientifique avant de s'impliquer dans les tâches d'écriture à planifier. Elles participent à alimenter la phase d'autorégulation que Pintrich (2004) appelle « l'anticipation et la planification». Les compétences sont classées ci-dessous par ordre croissant de compétence autodéclarée. Elles sont mises en regard d'une terminologie usuelle en langues et d'extraits de discours des étudiants qui s'auto-qualifient. 
Tableau 3 . Analyse des discours auto-qualifiants des étudiants sur leurs compétences en écriture scientifique en anglais avant le cours

\begin{tabular}{|l|l|l|}
\hline $\begin{array}{l}\text { Niveau } \\
\text { compétence }\end{array}$ & $\begin{array}{l}\text { Terminologie } \\
\text { courante }\end{array}$ & Discours hétérogènes d'étudiants \\
\hline Moindre compétence & Débutant & $\begin{array}{l}\text { J'écrivais très peu donc niveau pas très élevé } \\
\text { Médiocre }\end{array}$ \\
\hline $\begin{array}{l}\text { Compétence } \\
\text { intermédiaire/ }\end{array}$ & Intermenne & $\begin{array}{l}\text { Je juge mon niveau en anglais écrit comme moyen, } \\
\text { avant le cours d'Anglais scientifique. Cela faisait un } \\
\text { moment que je n'avais pas pratiqué }\end{array}$ \\
\hline $\begin{array}{l}\text { Moyen - environ B1 } \\
\text { Lacunes } \\
\text { Assez bon, intermédiaire }\end{array}$ \\
\hline Meilleure \\
compétence
\end{tabular}

Les étudiantes se situent sur un continuum de novice à expérimenté. Certaines ont plus de dix ans d'expérience en anglais (pour ceux qui en ont commencé l'apprentissage en première année d'école primaire et l'ont continué jusqu'à obtenir un diplôme de licence). D'autres se déclarent débutantes en écriture en anglais, ce qui peut refléter une réalité lorsque l'on vient de certains pays (l'Iran par exemple), ou bien signifier qu'il n'est rien resté des précédents apprentissages et que l'étudiant pense partir de zéro. Parmi les formulations choisies par les étudiants, on remarque l'usage d'une terminologie de jugement: «bon, moyen, médiocre, pas très élevé, intermédiaire, améliorable, correct, élevé » qui qualifie globalement un niveau, avec plus ou moins de

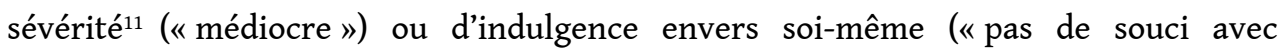
l'anglais »). La compétence est évaluée plus souvent en termes de niveau qu'en termes de savoirs ou de savoir-faire. Quelques étudiants emploient les codes de classification du CECRL (ex : B1, B2) d'autres se basent sur leur expérience et pratique.

Ces réponses nous informent sur le fait que les besoins d'aide sont variables. Ce résultat valide la proposition d'aides diversifiées ou pour des apprentissages diversifiés faite dans le cours. En creux, ces auto-évaluations étudiantes n'indiquent guère de mobilisation de connaissances spécifiques sur les contraintes de l'écriture scientifiques en anglais, qu'il faudrait construire et mobiliser pour mieux réussir dans le travail demandé. 


\subsection{Aide à l'implication dans la tâche et à l'accrochage}

41 Pour aborder le thème de l'aide à l'implication et à l'accrochage nous nous nous intéressons aux réponses à une question d'évaluation quantifiée des qualités du cours sous forme d'une échelle de Likert à 5 points (de 1 " pas du tout » à 5 « très »), à partir de 8 critères positifs. Les scores représentent la moyenne des points accordés à chaque critère. Tous les critères obtiennent un score supérieur à la notre moyenne de 2,5 dans l'ordre croissant suivant: (1) Facile : 2,8/5, (2) Concret: 3,2/5, (3) Motivant : 3,4/5, (4) Professionnalisant : 3,5/5, (5) Agréable : 3,7/5, (6) Important : 3,8/5, (7) Intéressant : $3,8 / 5,(8)$ Utile $: 3,9 / 5$.

La note la plus basse porte sur le critère de facilité et la note la plus haute sur le critère d'utilité. Les scores positifs $(>2,5)$ enregistrés par tous les critères nous encouragent à penser que dans l'ensemble cette expérience a été vécue de manière positive. D'après leur réponses, les étudiantes semblent avoir pu créer un lien entre le travail d'écriture académique demandé (avec les critères facile, motivant, agréable, intéressant) dans le cadre du cursus et basé sur des connaissances, et un enjeu professionnel défini par des compétences (avec les critères concret, professionalisant, important, utile). Nous en déduisons indirectement et en creux que les modalités d'aide ont été suffisantes pour soutenir l'implication et l'accrochage dans la tâche, ou pour lever les entraves ou résoudre les problèmes, notamment liés à l'anxiété engendrée par la situation d'autorégulation et d'autoévaluation. En effet, ces processus en auto déclenchent des émotions positives ou négatives tout au long des différentes phases de travail, provoquant soit une implication dans la tâche soit un renoncement (Fatoux, 2019).

\subsection{Aide à la conscientisation du développement de compétences articulées à des savoirs}

Interrogés sur l'évolution de leur sentiment de compétence une majorité d'étudiants déclarent mieux maitriser non seulement l'écrit scientifique en anglais, mais aussi les écrits scientifiques en général. Sur une échelle de Likert allant de 1 (je me sens moins compétent qu'avant le cours) à 5 (je me sens plus compétent qu'avant le cours), les étudiants doivent situer leur progrès en rédaction en anglais $(3,5 / 5)$, en apprentissage de langues en général $(3,6 / 5)$, en rédaction scientifique en anglais $(3,6 / 5)$ et en rédaction scientifique en général $(3,8 / 5)$. La majorité des étudiants déclare avoir progressé dans ces quatre compétences qui s'articulent avec des savoirs linguistiques et académiques, et surtout en rédaction scientifique en général, c'est-à-dire pas seulement en anglais.

Certes le sentiment de compétence n'est pas synonyme de compétence effective et on peut avoir le sentiment d'être compétent sans l'être, ou l'être pour une situation donnée sans certitude de transfert. En outre, ces données sont déclaratives et méritent une investigation plus poussée. C'est donc avec beaucoup de prudence que nous avançons ici que ce résultat pourrait être un indicateur de l'efficience des aides proposées. Au moins l'organisation n'a-t-elle pas eu pour résultats la régression ou la stagnation des compétences en question. Par ailleurs, une étude complémentaire pourrait chercher à obtenir des indications quant aux compétences développées dans l'autorégulation et la réflexivité. Un autre protocole méthodologique serait alors nécessaire. 


\subsection{Aide à une approche variée des apprentissages}

Dans cette section nous présentons l'avis des étudiants sur leur facilité ou difficulté à s'autoréguler à partir d'une liste de tâches qui font partie de la compétence d'écriture en anglais scientifique. Nous questionnons les étudiantes sur leur sentiment d'habileté dans ces tâches spécifiques parce qu'à chacune d'elle correspondent une ou plusieurs modalités d'aide dans le cours (voir Tableau 1). Il s'agit de classer chaque tâche selon le degré de facilité ou de difficulté perçu, sur une échelle de Likert à 5 points. Plus la note est haute, plus la tâche est perçue comme difficile. Les moyennes des réponses se répartissent comme suit, du moins difficile en haut, au plus difficile en bas : (1) Gérer le stress :2,6/5, , (2) Planifier vos actions: 2,8/5, (3) Comprendre les sources:3,0/5, (4) Mobiliser vos connaissances en langue anglaise :3,2/5, (5) Contrôler le projet :3,2/5, (6) Réviser votre texte : 3,4/5, (7) Mobiliser vos connaissances sur le sujet choisi : 3,5/5, (8) Mettre vos idées en texte :3,5/5, (9) Trouver des sources :3,6/5.

51 Toutes ces tâches reçoivent une note de difficulté supérieure à la note moyenne de $2,5 / 5$, et allant de 2,6 à 3,6/5. Le traitement de la situation d'écriture en anglais exige 
effectivement que les étudiantes se conforment à des contraintes liées à l'écriture d'un texte scientifique en anglais académique. Or ces contraintes semblent avoir été perçues comme des difficultés à surmonter. Cela nous permet d'interroger la part de la place de l'apprentissage dans l'action et celle de l'usage de savoirs pour réussir (Rey, 2014) et attire notre attention sur l'importance d'aider les étudiants à mieux faire ce lien entre les connaissances à mobiliser dans la tâche, et la réussite de la tâche. Des améliorations des modalités d'aide sur ce point pourraient être envisagées. Il est par ailleurs possible que ce choix de réponse soit une stratégie pour éveiller l'indulgence de l'enseignante vis-à-vis des productions finales et mettre en valeur l'effort fourni malgré la difficulté.

\section{Conclusion}

L'organisation que nous avons imaginée, expérimentée et évaluée, se révèle non seulement adaptée, mais aussi adaptable, aux contraintes de notre terrain et de notre public diversifié. Elle apporte une réponse concrète qui prend en compte le problème de l'hétérogénéité langagière des étudiants. Elle est cohérente avec les contenus de discipline et de professionalisation du master. Cette organisation, nous l'avons constaté et fait évaluer par les étudiants, optimise le temps réel dédié à l'acte d'écriture de recherche dont une grande partie, il ne faut pas l'oublier, est consacrée à la recherche, à la lecture et à la réflexion. Elle peut donc être une réponse au besoin de différenciation pédagogique dont l'université a besoin dans le contexte de massification de ses publics.

Les étudiantes ont eu en majorité le sentiment d'avoir progressé en rédaction scientifique en anglais et en français. En revanche, mobiliser les connaissances nécessaires pour gérer en autonomie les contraintes liées aux écrits scientifiques en anglais a été vécu comme difficile (mais qui a dit que les études en master devaient être faciles?). Ce résultat nous invite à réfléchir à une amélioration de l'aide visant ces connaissances et leur mobilisation dans la tâche attendue. Une évaluation spécifique des modalités visant cet objectif pourrait faire l'objet d'une autre recherche à partir d'un nouveau protocole de recherche intégrant des entretiens. Quant à savoir si, et en quoi, les compétences à s'auto-réguler dans le processus d'écriture et les compétences en anglais scientifique écrit ont évolué, d'autres investigations s'avèrent également nécessaires. Les contenus des carnets de bord étudiants pourraient contenir des indications utiles à ce sujet.

Nos résultats montrent que le système d'aide semble efficient en ce qui concerne l'implication et l'accrochage à la tâche. Il met en lumière la préférence des étudiantes pour les modalités d'aide qui impliquent activement l'enseignante dans un dialogue autour du projet, via le conseil synchrone en classe, et asynchrone en articulation avec le carnet de bord. Si la pandémie Covid-19 qui sévit en 2020 rend tentante l'imposition du mode distancie pour l'enseignement, il est intéressant de remarquer que, chez nos étudiantes, c'est la présence enseignante, qu'elle soit physique ou médiatisée, qui est plébiscitée comme le meilleur soutien à leur travail académique. 


\section{BIBLIOGRAPHY}

Albero, B. (2000). L'autoformation en contexte institutionnel. Du paradigme de l'instruction au paradigme de l'autonomie. L'Harmattan.

Abrams, Z. I. (2019). The effects of integrated writing on linguistic complexity in L2 writing and task-complexity. System, 81, 110-121. https://doi.org/10.1016/j.system.2019.01.009

Amadieu, F. et Tricot A. (2015), Les facteurs psychologiques qui ont un effet sur la réussite des étudiants. Recherche et pratiques pédagogiques en langues de spécialité, XXXIV(2). http:// journals.openedition.org/apliut/5155

Bailly, S. (1995). La formation de conseiller. Mélanges CRAPEL, (22), 63-83.

Bailly, S. et Ciekanski, M. (2004, 2 avril). Le SAAS (système d'apprentissage auto-dirigé avec soutien) du CRAPEL : nouveaux dispositifs et nouveaux rôles pour l'apprentissage des langues autrement. Dans Le FLE en pratique(s). Cinquième Journée interrégionale de l'AREFLE, Université Marc Bloch. Strasbourg, France.

Bandura, A. (1991). Social cognitive theory of self-regulation. Organizational Behavior and Human Decision Processes, 50, 248-287. http://www.des.emory.edu/mfp/Bandura19910BHDP.pdf

Barré de Miniac, C. (2001). Le rapport à l'écriture. Aspects théoriques et didactiques. Presses universitaires du Septentrion.

Blašková, M. (2014). Influencing Academic Motivation, Responsibility and Creativity. Procedia Social and Behavioral Sciences, (159), 415-425.

Bonnassies, I. (2006) L'apport des TICE et de l'écriture collaborative au développement des compétences écrites dans l'acquisition/apprentissage d'une L2. Cahiers de l'APLIUT, XXV(3), 42-59.

Carré, P. et Moisan, A. (2002). La formation autodirigée: aspects psychologiques et sociologiques. L'Harmattan.

Carette, E. et Holec, H. (1995). Quels matériels pour les centres de ressources. Mélanges CRAPEL, (22), 85-94.

Chateau, A. et Zumbihl, H. (2010). Le carnet de bord, un outil permettant le cheminement vers l'autonomisation dans un dispositif d'apprentissage de l'anglais en ligne? Alsic, 13, http:// journals.openedition.org/alsic/1392

Ciekanski, M. (2005). L'accompagnement à l'autoformation en langue étrangère : contribution à l'analyse des pratiques professionnelles (Étude des dimensions langagières et formatives des pratiques dites « de conseil » dans des systèmes d'apprentissage autodirigé en langue étrangère) [thèse de doctorat, Université Nancy 2]. https://tel.archives-ouvertes.fr/tel-00398940

Ciekanski, M., Bailly, S., Chateau, A, Bary, R., Rees, M. et Candas, P. (2016). De l'expérience aux compétences en langues : le rôle des écrits réflexifs dans le processus d'autonomisation des étudiants. Dans Biarnes J. et Rose J. (dir.), Les portefeuilles d'expériences et de compétences. Approches pluridisciplinaires (p. 29-44). Presses universitaires du Septentrion.

Coffin, C, Curry, M. J., Goodman, S., Hewings, A., Lillis, T. M. et Swann, J. (2003). Teaching Academic Writing. A toolkit for higher education. Routledge.

Coleman J. A. (2014) How to get published in English : Advice from the outgoing Editor-in-Chief. System, 42, February, 404-411. https://doi.org/10.1016/j.system.2014.01.004 
Escorcia, D. et Fenouillet, F. (2011). Quel rôle de la métacognition dans les performances en écriture? Analyse de la situation d'étudiants en sciences humaines et sociales. Canadian Journal of Education/Revue canadienne de l'éducation, 34(2), 53-76. http://www.jstor.org/stable/ canajeducrevucan.34.2.53

Fatoux, C. (2019). L'autorégulation dans les demandes d'aide d'étudiants universitaires de premier cycle dans un cours en ligne [thèse de doctorat, Université Laval]. Corpus. http://hdl.handle.net/ 20.500.11794/35741

Gremmo, M-J. (1995). Conseiller n'est pas enseigner. Le rôle du conseiller dans l'entretien de conseil. Mélanges CRAPEL, (22) 33-61.

Halté, J.-F. (1989). Savoir écrire - savoir faire. Pratiques : linguistique, littérature, didactique, (61), 3-28. https://doi.org/10.3406/prati.1989.1500

Holec, H. (1981). Autonomy and foreign language learning. Ergamon.

Holec, H. (1988). Autonomie et Apprentissage auto-dirigé. Conseil de l'Europe.

Holec, H. (1990). Des documents authentiques pour quoi faire? Mélanges Pédagogiques, 65-74. http://www.atilf.fr/IMG/pdf/melanges/5holec-2.pdf

Holec, H. (1998). L'apprentissage autodirigé : une autre offre de formation. Le français dans le monde recherches et Applications, Apprentissage des usages des langues dans le cadre européen, (numéro spécial), 213-255.

Giveh, F., Ghobadi, M. et Zamani, Z. (2018) Self-directed Learning in L2 Acquisition : A Review of Theory, Practice, and Research. Journal of Language Teaching and Research, 9(6), 1335-1343.

Hayes, J. R. et Flower, L. S. (1980). Identifying the organization of writing processes. Dans L. W. Gregg et E. R. Steinberg (éd.), Cognitive processes in writing. Lawrence Erlbaum.

Howes, D. (1999). A response to « Academic literacy: a case study ». Dans C. Jones, J. Turner et B. Street (éd.), Student writing in the university : Cultural and epistemological issues (p. 207-210). John Benjamins.

Johns, A. M. (1997). Text, role, and context : Developing academic literacies. Cambridge University Press.

Lochard, Y. (2007). L'avènement des « savoirs expérientiels ». La Revue de l’Ires, 55(3), 79-95

Macrae, M. (1997). The induction of international students to academic life in the United Kingdom. Dans D. McNamara et R. Harris (éd.), Overseas students in higher education: Issues in teaching and learning (p. 139-142). Taylor \& Francis.

Pajares, F. (2003). Self-efficacy beliefs, motivation, and achievement in writing : a review of the literature. Reading \& Writing Quarterly, (19), 139-158.

Pasek, J. (2012). Writing the Empirical Social Science Research Paper : A Guide for the Perplexed. Psychology Teacher Network. http://www.apa.org/education/undergrad/empirical-socialscience.pdf

Paul, M. (2004). L'accompagnement : une posture professionnelle spécifique. L'Harmattan.

Pintrich, P. R. (2004). A Conceptual Framework for Assessing Motivation and Self Regulated Learning in College Students. Educational Psychology Review, 16(4), 385-407.

van Enka, A. et Power, K. (2017) What is a research article? Genre variability and dataselection in genre research. Journal of English for Academic Purposes, 29, September, 1-11. 
Rey, B. (2014). La notion de compétence en éducation et en formation. Enjeux et problèmes. De Boeck.

Street, B. (1999). Academic literacies. Dans C. Jones, J. Turner et B. Street (éd.), Student writing in the university: Cultural and epistemological issues (p. 193-199). John Benjamins.

Van de Poel, K. et Gasiorek, J. (2012). Effects of an efficacy-focused approach to academic writing on students' perceptions of themselves as writers. Journal of English for Academic Purposes, 11, 294-303.

Xiao, Y. et Yang, M. (2019). Formative assessment and self-regulated learning : How formative assessment supports students' self-regulation in English language learning. System, 81, 39-49. https://doi.org/10.1016/j.system.2019.01.004

\section{NOTES}

1. Le $\mathrm{SE}=$ le savoir écrire. Note insérée par l'autrice de cet article.

2. Le SF = le savoir-faire. Note insérée par l'autrice de cet article.

3. Arche est le nom de la plateforme Moodle de l'UL.

4. European Credits Transfer System ou système européen de transfert et d'accumulation de crédits en français.

5. Le choix du sujet est libre, ce qui dans certains cas, favorise l'engagement de l'étudiant dans son projet. Pour aider la réflexion des étudiants des pistes leurs sont proposées, sous forme de listes, qu'ils peuvent suivre ou non.

6. Ressource ajoutée en 2017 lors de la parution de l'article

7. En didactique des langues cette notion désigne des documents qui n'ont pas été produits à des fins d'enseignement-apprentissage mais de communication, contrairement aux documents dits didactiques (Holec 1990)

8. Par exemple, avec le français comme langue d'interface : http://fcorpet.free.fr/Denis/Redigermanuscrit.html et https://blog.espci.fr/laurette/anglais-pour-scientifiques-francais/; https:// www.grenoble-sciences.fr/pap-ebooks/upjohn/minimum-competence-scientific-englishet; avec l'anglais en langue d'interface : http://www.phrasebank.manchester.ac.uk/introducing-work/;

9. Notons que sur ce point de la notation chiffrée le contrôle bascule de l'étudiant à l'enseignante qui a le dernier mot. Ceci est peut-être une limite au projet d'émancipation sous-jacent aux approches autodirigées ou autonomisantes. Mais l'institution universitaire est-elle prête à se passer de notes chiffrées ou à valider des notes auto-administrées par les étudiants sans les contrôler?

10. Incidemment, les contenus du questionnaire peuvent les aider à améliorer leurs manières de faire en leur présentant des stratégies à essayer.

11. Peut être comme stratégie de mise en valeur du résultat final.

\section{ABSTRACTS}

To cope with the growing diversity and linguistic heterogeneity of the student population at the University of Lorraine (UL), a self-directed English for (written) Academic Purposes course was designed in 2014 for the Master's students of Didactique des langues/fle. Students can make 
progress at their own pace and benefit from personalised support. To complete this course, the students must write a long scientific text in English, making use of self-regulated practices and a variety of resources made available to them on the UL course platform. These resources support, on the one hand, the building of new knowledge and skills needed to successfully complete the required task, and on the other hand, learner autonomy and self-regulation steeped in cognitive and affective dimensions. For the purposes of this study, we will analyse the design of the support system offered as well as its effects, by making use of assessment questionnaires provided at the end of the semester. Results indicate that although the students found the course difficult, the efficiency of the aid to support their engagement and adherence to the required task, which are important ingredients of self-regulation. Among the different kinds of aids offered, students prefer those that involve direct oral or written interaction with the teacher, such as one-to-one advisory sessions and the logbook.

Etant donnée la diversité croissante des publics de l'université et leurs hétérogénéités linguistiques et langagières, le cours d'anglais scientifique du master didactique des langues/fle de l'Université de Lorraine (UL) se présente depuis 2014 sous la forme d'un dispositif d'apprentissage autodirigé avec soutien. Les étudiants peuvent aller à leur rythme et bénéficier d'un accompagnement personnalisé. Pour valider ce cours, les étudiants doivent rédiger un texte scientifique long en anglais dans leur discipline, de manière autorégulée et à l'aide d'une variété de ressources mises à leur disposition en appui sur la plateforme de cours de l'UL. Ces ressources sont censées d'une part aider la construction autonome de connaissances et de compétences qui s'articulent dans l'acte d'écriture scientifique en anglais, et d'autre part, soutenir le processus d'auto-régulation et d'autonomisation dans ses dimensions cognitives et affectives. Pour cette recherche, nous analysons le design du système d'aide proposé, ainsi que ses effets à partir de questionnaires d'évaluation du cours proposés en fin de semestre. Les résultats indiquent que les étudiants ont trouvé ce cours difficile mais que les aides à l'implication et l'accrochage à la tâche demandée, ingrédients importants de l'autorégulation, ont été efficientes. Parmi les modalités d'aide proposées, les préférées sont celles qui impliquent une interaction directe entre l'enseignante et les étudiants à l'oral et à l'écrit, comme l'entretien de conseil et le journal de bord.

\section{INDEX}

Mots-clés: aide à l'apprentissage; rédaction scientifique en anglais lansad; apprentissage autodirigé avec soutien

\section{AUTHORS}

\section{SOPHIE BAILLY}

Equipe Didactique des langues et sociolinguistique, Université de Lorraine

\section{VÉRONIQUE LEMOINE-BRESSON}

Equipe Didactique des langues et sociolinguistique, Université de Lorraine 